\title{
MULTISCALE BEHAVIOR OF A SIMPLE MODEL FOR STOCK MARKETS
}

\author{
JUAN R. SÁNCHEZ
}

Received 15 December 2004

The multiscale behavior of a recently reported model for stock markets is presented. It has been shown that indexes of real-world markets display absolute returns with memory properties on a long-time range, a phenomenon known as cluster volatility. The multiscale characteristics of an index are studied by analyzing the power-law scaling of the volatility correlations which display nonunique scaling exponents. Here such analysis is done on an artificial time series produced by a simple model for stock markets. After comparison, excellent agreements with the multiscale behavior of real-time series are found.

\section{Introduction}

There seems to be a general agreement on the statement that stock market's absolute returns (see below for definition) have long-range correlation properties, a phenomenon known in financial literature as cluster volatility. More recently, a scaling analysis of the generalized cumulative absolute returns has been done, showing that volatility correlations are power-law correlated on a long-time range (from days to one year) and that the correlation exponent is not unique, a phenomenon known in the theory of dynamical systems as multiscaling [4].

On the other hand, several computational models trying to represent the behavior of actual stock markets have been presented $[6,7,8,9]$. From a theoretical point of view, it is clear that it could be very useful to have available a model that could reproduce as closely as possible the behavior of real-world markets. Here a multiscaling analysis on the time series generated by a recently reported model for stock markets is presented [6]. It is found that the scaling properties of the artificial time series generated by the model are in excellent agreement with those of actual time series.

In the rest of this introductory section, a brief description of the model used to generate the time series is presented. A complete description of the model as well as other characteristics can be found in [6]. The theory and methods for analyzing the properties of a time series in searching for multiscale behavior are outlined in Section 2 as well as the results of the present study. 
1.1. Model. In order to obtain a good representation of the time evolution of actual markets, two types of "traders" must be included in a model: the followers and the fundamentalists. The so-called followers are supposed to follow the local (in space and time) trend of the market, buying or selling orders on a given stock following the behavior of other (related) stocks. They are also called noisy traders. On the other hand, there are also traders who are considered to be responsible for the market turnoffs. These kinds of traders are supposed to know something more about the market and then, are able to develop some kind of more sophisticated strategy to operate. They can take actions to buy or sell according to other indicators; the market's fundamentals. These indicators could depend on other types of information which usually come from outside of the market. It is more realistic to think that both types of behaviors are acting at the same time and influence the way in which a specific stock price changes.

In the model of [6], the market is modeled by a vector of stock prices $\mathbf{x}$ having $N$ integer-valued components $x_{i}$, each one representing the price of a market asset (in arbitrary units). Then, associated with each $x_{i}$ is a direction of movement value $v_{i}$. The components of the direction of movements vector $\mathbf{v}$ are of Ising type, that is, they can take two values $v_{i}= \pm 1$. The model evolves in time according to the following dynamical rules. The evolution of a (randomly chosen) component $x_{i}$ follows the equation

$$
x_{i}(t+\Delta t)=x_{i}(t)+v_{k}(t)
$$

while for the corresponding components $v_{i}$, the following evolution equation is valid:

$$
v_{i}(t+\Delta t)= \begin{cases}v_{i \pm 1}(t) & \text { if }\left|x_{i}(t)\right|<X_{t h} \\ -v_{i}(t) & \text { if }\left|x_{i}(t)\right|>X_{t h}\end{cases}
$$

The value of $v_{i}(t+\Delta t)$ is obtained by choosing at random among the direction values of each one of the neighbors, that is, $v_{k}=v_{i-1}$ or $v_{k}=v_{i+1}$ with equal probability. In principle, the algorithm described by the equations above takes into account the influence of the noisy traders who follow the trend of related stocks in order to buy $\left(v_{i}=+1\right)$ or to sell $\left(v_{i}=-1\right)$ a specific asset. However, it is not reasonable to think that the prices $\mathbf{x}$ could take arbitrary positive or negative values. There is no actual market following a given trend for ever. Then in order to take into account the influence of the fundamentalist traders, a threshold $X_{t h}$ is established for the absolute value of each $x_{i}$. As it can be seen in (1.2), if at any time $\left|x_{i}\right|>X_{t h}$, the corresponding direction of movement $v_{i}$ is reversed, $v_{i} \rightarrow-v_{i}$. This reversal procedure simulates the influence of the fundamentalist traders who, when the absolute value of a stock reaches a value $X_{t h}$, consider that the price is low enough so it is time to buy or it is high enough and then it is time to sell.

A representative index for the described artificial market is taken to be the mean value time series

$$
x_{M}(t)=S(t)=\frac{1}{N} \sum_{i=1}^{N} x_{i}(t) .
$$




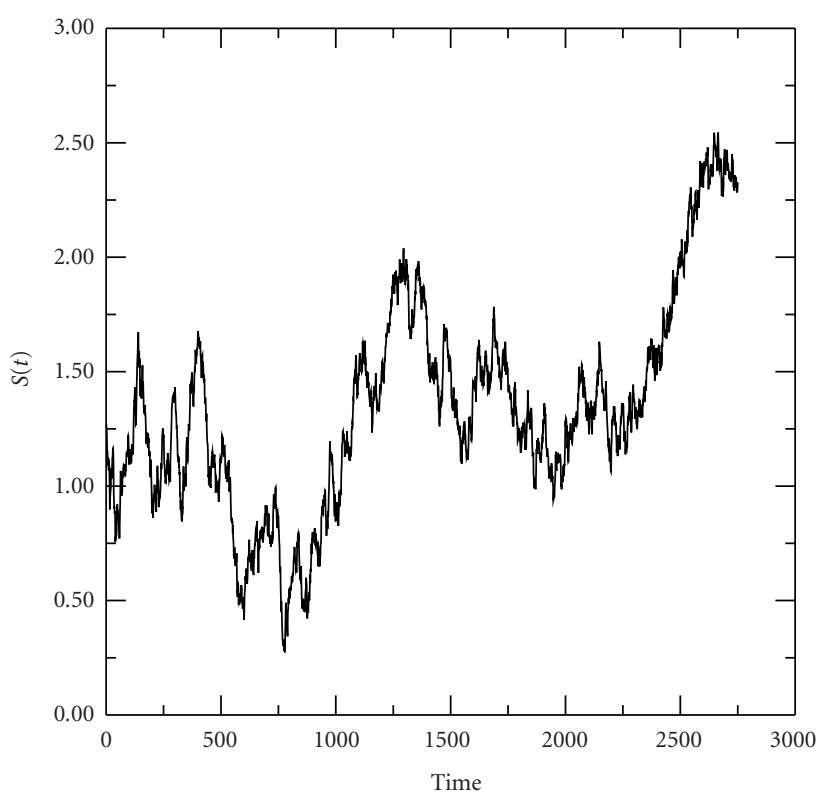

Figure 1.1. A typical path of the simulated price process $S(t)$ up to $T=2750$. The data of this figure are used in the multiscaling analysis.

Here, the corresponding returns are defined as

$$
R(t)=\frac{|S(t+1)-S(t)|}{|S(t)|}
$$

where $|\cdot|$ represents the absolute value operation on the argument. Because in the abovepresented model, prices $S(t)$ are in arbitrary units, it is necessary to use absolute values in order to avoid negative arguments within the detrended logarithm of returns used for the multiscaling analysis (see (2.2) below).

The dynamical behavior of the above-described model was investigated in [6] by means of Monte Carlo simulations. $N=1024$ and $X_{t h}=30$ where used as typical parameters. Using these parameters, a typical path of the simulated price $S(t)$ up to $T=2750$ is shown in Figure 1.1. The total amount of data was arbitrarily taken to be small in order to reproduce as close as possible the conditions of a real market study. Although the statistical properties reported here have been verified to be consistent with several time series obtained from different simulations using the same set of parameters, the $S(t)$ data presented in Figure 1.1 were used in the multiscaling study. These data are available from the author upon e-mail request in the form of a two-column ASCII file representing $t$ and $S(t)$.

\section{Multiscale analysis and results}

We consider at first if some general statistical property of the probability distribution function (pdf) of the artificial series under study is similar to the other found in real-world 
time series. In particular, it was pointed out by several authors that the distribution of returns is leptokurtic [2]. This property was analyzed in [1] by considering the distance $h$ between the empirically calculated pdf of a random variable $X$ and the Gaussian distribution. The quantity $h$ is defined as

$$
h \equiv \frac{\langle x\rangle}{\sqrt{\left\langle x^{2}\right\rangle-\langle x\rangle^{2}}} .
$$

It is nondimensional and it takes the value $h_{G}=\sqrt{2 / \pi}$ for the Gaussian pdf. For other leptokurtic pdf's (such as Laplace distribution), $h$ is always smaller than $h_{G}$ and it is considered to be a good parameter to measure the degree of leptokurtosis of a pdf [1].

Here, the quantity $h$ was calculated for the artificial time series of returns $R(t)$ (see (1.4)) in two forms, global and local. A global value of $h$ for the whole time series was calculated by applying directly (2.1) to all the 2750 values available. It results in a global value for the relation $h / h_{G}$ of 0.87 , a clear indication of a leptokurtic pdf. A second test was done locally (in time) by dividing the $R(t)$ series into $T / \tau$ nonoverlapping segments, with $\tau$ being the length of each segment.

In general, the $\tau$ is taken to be a representative time interval on which a given property is expected to have an interesting behavior. It can go from one "day," $\tau=\Delta t=1$, to one "year" of trading, $\tau=\Delta t=250$. The values of $R(t)$ within each segment were considered as independent time series and (2.1) was applied to each one of them. The values of $h$ for intervals of length $\tau=200$ are shown in Figure 2.1. It can be seen that, mostly, the behavior of $R(t)$ is leptokurtic. This is a first indication that the artificial time series under study represents very well an actual market (or stock) index.

In order to start the multiscaling study, following [4], a quantity named the detrended logarithm of returns is defined as

$$
r(t)=\log R(t)-\langle\log R(t)\rangle
$$

where $R(t)$ is the value of the return at time and $\langle\cdot\rangle$ represents the average over the whole sequence. The underlying volatility $\sigma_{t}$ is usually defined as $r_{t}=\sigma_{t} \eta_{t}$, where $\eta_{t}$ are identically distributed random variables. Once the log returns are defined, the set of variables called cumulative returns

$$
\rho_{t}(\tau)=\frac{1}{\tau} \sum_{i=0}^{\tau-1} r_{t+i}
$$

is considered. From the assumption that $r_{t}$ are uncorrelated in the long range, it follows that the standard deviation $\sigma(\tau)$ of the nonoverlapping variables $\rho_{t}(\tau)$ should have a power-law behavior of the type $\sigma(\tau) \sim \tau^{-\beta}$ with $\beta \simeq 0.5$. In Figure 2.2, the standard deviation of the variables $\rho_{t}(\tau)$ is $\log$-log plotted against $\tau$ for the returns calculated using (2.2) from the index data of Figure 1.1. The slope of a line through the slanted crosses gives the exponent $\beta=0.55$. A value of $\beta$ close to 0.5 is an indication that the cumulative returns are not correlated in the long range. A similar characteristic was found for the NYSE index $[3,4]$. 


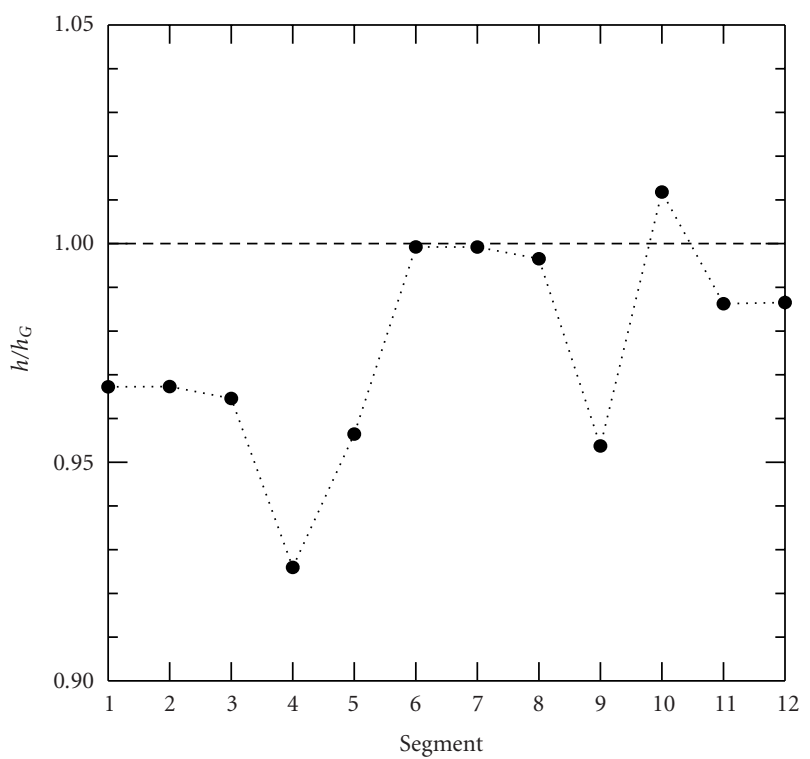

Figure 2.1. Plot of the relation $h / h_{G}$ calculated independently for twelve segments of length $\tau=200$. Most of the points are under the $h / h_{G}=1$ line, indicating a leptokurtic characteristic of each time series.

In order to study the correlations of the absolute values of the returns, the generalized cumulative absolute returns

$$
\varrho_{t}(\tau, \gamma)=\frac{1}{\tau} \sum_{i=0}^{\tau-1}\left|r_{t+i}\right|^{\gamma}
$$

are introduced. $\gamma$ is a real-valued exponent and if the absolute returns happen to be uncorrelated, again an exponent $\beta \simeq 0.5$ for the scaling of the standard deviations should be obtained no matter which value of $\gamma$ is used in the calculations of (2.4). In particular, the signature of the multiscale behavior of a time series is the multiscaling power-law behavior of the correlation through the relation $\beta(\gamma)=\alpha(\gamma) / 2$, with $\alpha(\gamma)$ being the scaling exponent of the autocorrelation function

$$
C(\tau, \gamma)=\left\langle\left|r_{t}\right|^{\gamma}\left|r_{t+\tau}\right|^{\gamma}\right\rangle-\left\langle\left|r_{t}\right|^{\gamma}\right\rangle\left\langle\left|r_{t+\tau}\right|^{\gamma}\right\rangle \sim \tau^{-\alpha(\gamma)}
$$

In Figure 2.2, two other plots are included. They show the dependence of the standard deviation $\varsigma(\tau, \gamma)$ of the variables $\varrho_{t}(\tau, \gamma)$ as a function of $\tau$, for $\gamma=1.0$ and $\gamma=3.0$. It can be seen that there is a dependence of the exponent $\beta$ on $\gamma$ which denotes the presence of different scales. Finally, this result is confirmed by the plot of Figure 2.3 in which the variation of the exponent $\beta$ on $\gamma$ is presented in the range $0.1<\gamma<10$. The longest correlation is obtained for $\gamma \cong 0.75$, corresponding to $\beta \cong 0.11$. The plot of Figure 2.3 is the signature of the multiscale behavior of the time series since it implies the multiscaling power-law behavior of the correlations through the above-mentioned relation between $\beta(\gamma)$ and $\alpha(\gamma)$. 
116 Multiscale behavior of a simple model for stock markets

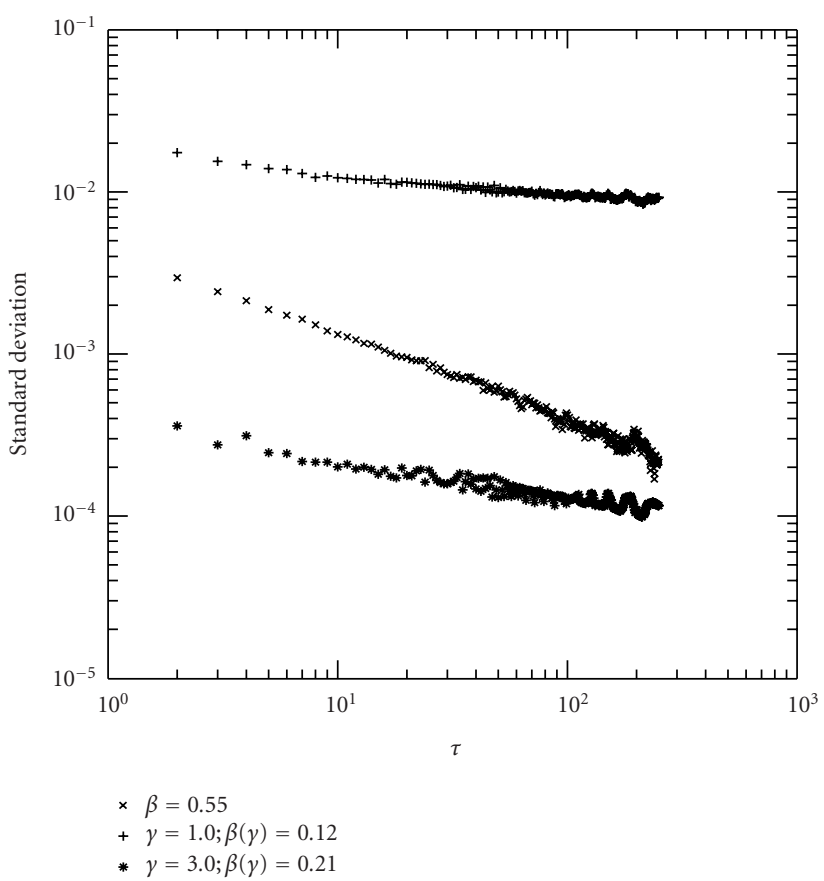

Figure 2.2. Dependence of the standard deviations $\sigma(\tau)(\times \operatorname{symbol})$ and $\varsigma(\tau, \gamma)(+$ and $*$ symbols $)$ of the variables $\rho(\tau)$ and $\varrho_{t}(\tau, \gamma)$, respectively. $\varsigma(\tau, \gamma)$ is displayed for $\gamma=1.0$ and $\gamma=3.0$ showing the dependence of the slope on the exponent $\gamma$.

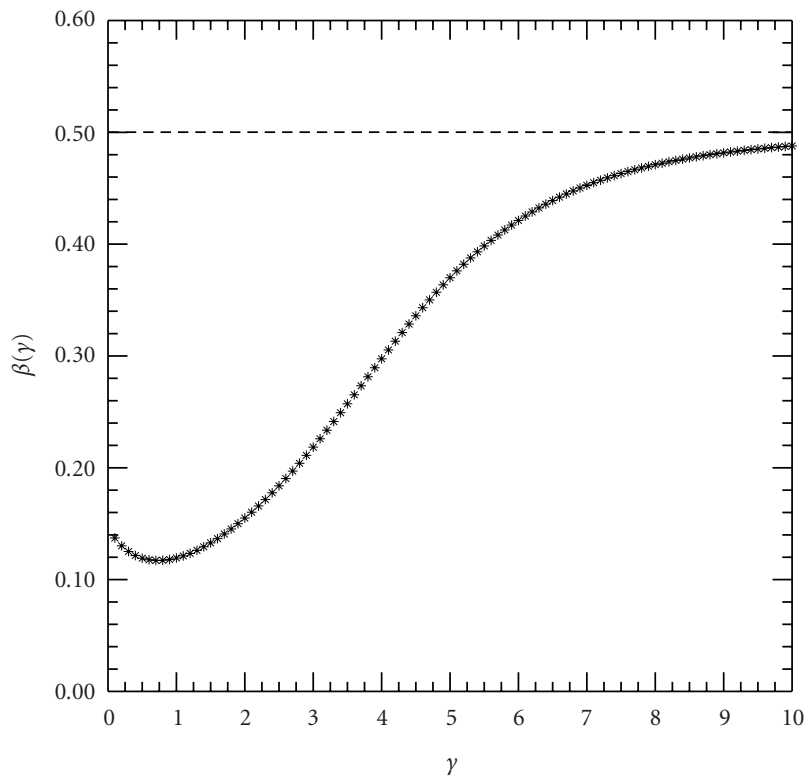

Figure 2.3. Variation of the scaling exponent $\beta$ as a function of $\gamma$ in the range $0.1<\gamma<10$. 


\section{Conclusions}

A multiscale analysis on an artificial time series generated by a model representing the behavior of a stock market has been presented. The results show that the simulated time series obtained from the operation of the dynamical system defined by (1.1) and (1.2) have the correct statistical properties to be considered as an excellent representation of the operation of an actual stock market. In particular, it has been shown that the simulated time series show multiscaling characteristics very similar to those recently reported for real-world time series $[4,5]$.

\section{References}

[1] F. Lillo and R. N. Mantegna, Variety and volatility in financial markets, Phys. Rev. E 62 (2000), no. 5, 6126-6134.

[2] R. N. Mantegna and H. E. Stanley, Scaling behaviour in the dynamics of an economic index, Nature 376 (1995), 46-49.

[3] - Turbulence and financial markets, Nature 383 (1996), 587-588.

[4] M. Pasquini and M. Serva, Multiscaling and clustering of volatility, Phys. A 269 (1999), no. 1, $140-147$.

[5] _ Clustering of volatility as a multiscale phenomenon, Eur. Phys. J. B 16 (2000), no. 1, 195-201.

[6] J. R. Sánchez, A simple model for stocks markets, Internat. J. Modern Phys. C 13 (2002), no. 5, 639-644.

[7] D. Stauffer, A. O. Sousa, and S. Moss de Oliveira, Generalization to square lattice of SZNAJD sociophysics model, Internat. J. Modern Phys. C 11 (2000), no. 6, 1239-1245.

[8] K. Sznajd-Weron and J. Sznajd, Opinion evolution in closed community, Internat. J. Modern Phys. C 11 (2000), no. 6, 1157-1165.

[9] K. Sznajd-Weron and R. Weron, A simple model of price formation, Internat. J. Modern Phys. C 13 (2002), no. 1, 115-123.

Juan R. Sánchez: Facultad de Ingeniería, Universidad Nacional de Mar del Plata, J.B. Justo 4302, 7600 Mar del Plata, Argentina

E-mail address: jsanchez@fi.mdp.edu.ar 


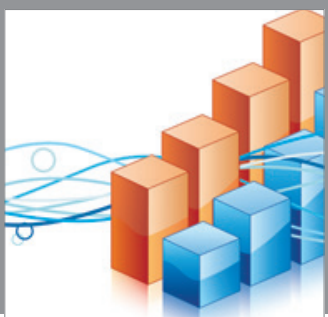

Advances in

Operations Research

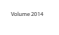

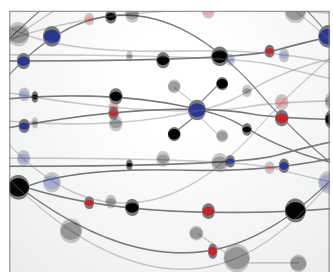

\section{The Scientific} World Journal
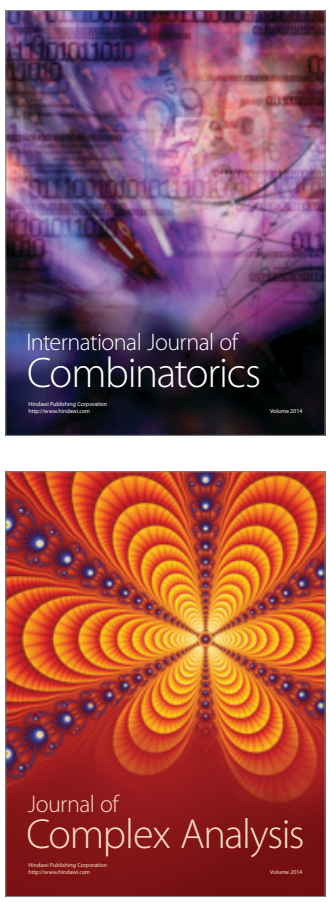

International Journal of

Mathematics and

Mathematical

Sciences
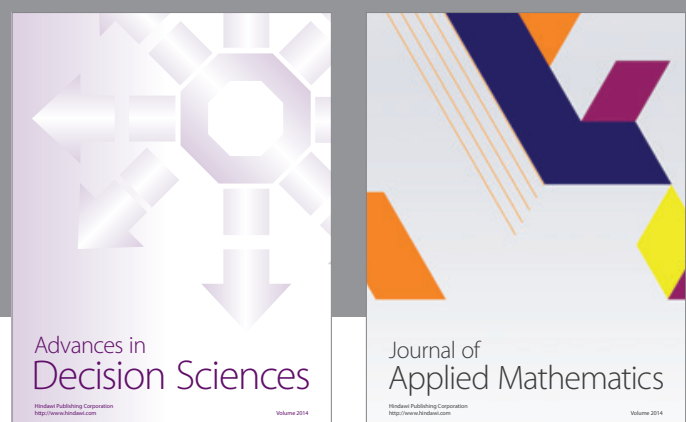

Journal of

Applied Mathematics
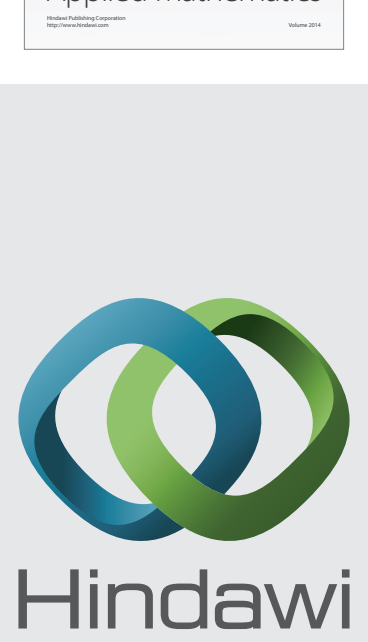

Submit your manuscripts at http://www.hindawi.com
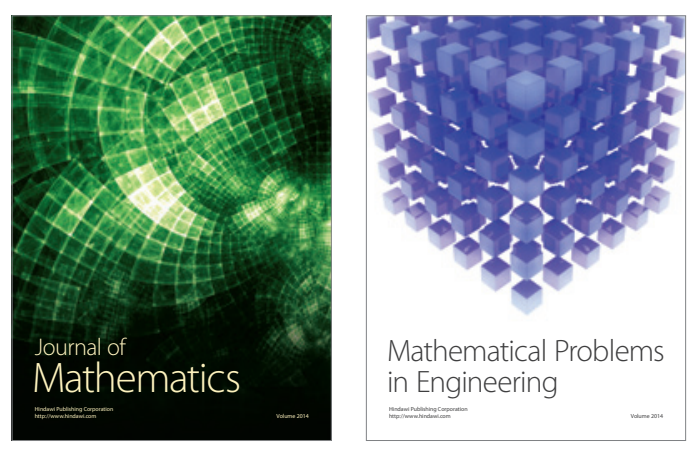

Mathematical Problems in Engineering
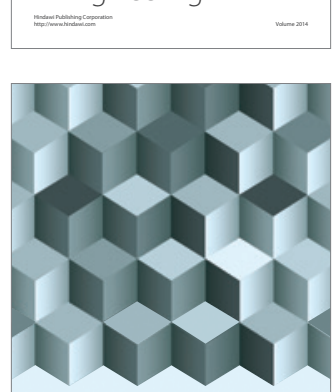

Journal of

Function Spaces
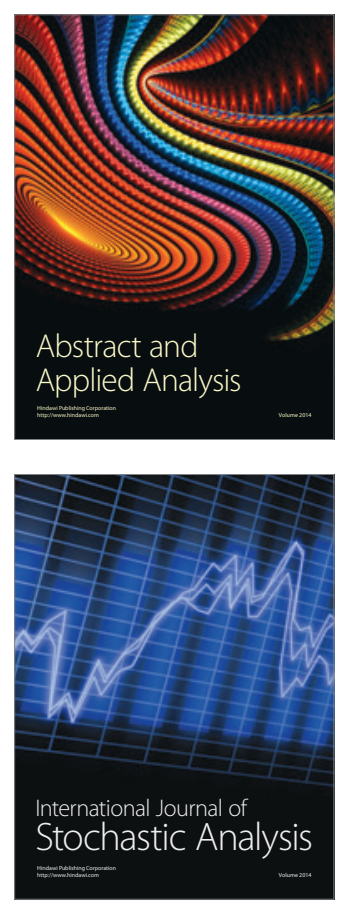

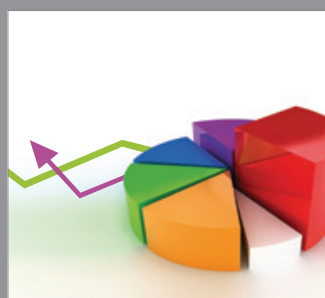

ournal of

Probability and Statistics

Promensencen
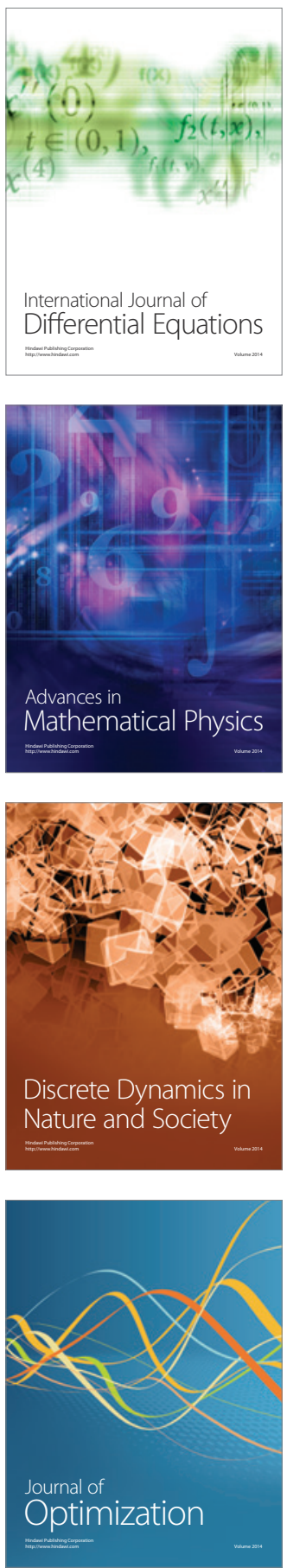